\title{
Application of Histochemical Stains for Rapid Qualitative Analysis of the Lignin Content in Multiple Wood Species
}

\author{
Yan Yang, ${ }^{\mathrm{a}}$ Yiming He, ${ }^{\mathrm{a}}$ Li Han, ${ }^{\mathrm{b}}$ Aifeng Wang, ${ }^{\mathrm{a}}$ Wei Wang, ${ }^{\mathrm{a}}$ Rui Zhao, ${ }^{\mathrm{a}}$ and Bin $\mathrm{Li}^{\mathrm{a}, *}$
}

\begin{abstract}
Rapid qualitative analysis was used to determine the influence of the lignin content of wood cell walls on the compression and bending properties of multiple wood species. The lignin type and cell wall content of Cunninghamia lanceolate, Fagus longipetiolata, Betula alnoides, Fraxinus mandshurica, and Tectona grandis was analyzed via histochemical staining, which included: the Mäule staining reaction, the Weisner staining reaction, and a fluorescence reaction. The results showed that the more red the Mäule staining reaction was, the greater the Syringyl lignin (S-type lignin) content was, and the more yellowish-brown the Mäule staining reaction was, the greater the Guaiacyl lignin (G-type lignin) content was. In addition, the more reddish-purple the Wiesner staining reaction was, the greater the lignin content was. The greater the brightness value of the fluorescence reaction was, the greater the lignin content was. Due to the negative correlation between the lignin content of the wood cell wall and the bending and compression properties of the wood, the application of histochemical stains for the analysis of wood lignin content could provide a reference and experimental basis for bending and compression treatments of various woods.
\end{abstract}

Keywords: Lignin content; Guaiacyl lignin (G-type lignin); Syringyl lignin (S-type lignin); Mäule staining reaction; Weisner staining reaction; Fluorescence reaction

Contact information: a: School of Architecture and Urban Planning, Nanyang Institute of Technology, Nanyang City, Henan Province, 473000, P.R. China; b: Henan Key Laboratory of Zhang Zhongjing Formulae and Herbs for Immunoregulation, Nanyang Institute of Technology, Nanyang City, Henan Province, 473000, P.R. China; * Corresponding author: yangyanrainy@163.com

\section{INTRODUCTION}

The wood cell wall is primarily composed of cellulose, hemicellulose, and lignins. As a basic unit of the wood cell wall, the lignin content has a strong influence on pulping and paper making processes, bending, and compression, etc. In general, the greater the lignin content, the greater will be the consumption of chemicals needed to remove the lignins from wood. This also means that a greater amount of energy generally is required to break the lignin polymers into small molecular structures during the softening process, making the process more difficult (Cui et al. 2016). In addition, the lignin content directly affects the degree of hardness for the wood. Wood with a higher lignin content usually is more difficult to bend or compress. The study of lignin content in multiple wood species could provide a preliminary reference and theoretical basis for the specific use of wood.

Lignins contain chromogenic groups, such as carbonyls, carboxyls, and alkenes conjugated with a benzene ring, and chromophore groups, such as phenolic hydroxyls and alcohol hydroxyls. Therefore, the lignin content could be qualitatively or quantitatively determined via a chromogenic or chromic reaction (Liu et al. 2004). The important colour reactions for the determination of lignin content were the Mäule staining reaction and the 
Wiesner staining reaction. The Mäule staining reaction primarily stains syringyl lignins (Stype lignins) in the wood cell wall into a red or reddish-purple, and it stains guaiacyl lignins (G-type lignins) to a yellow or yellowish-brown. The lignin type in softwoods is primarily the G-type lignin, while hardwoods contain both G-type lignins and S-type lignins (Liu et al. 2004). Therefore, the Mäule staining reaction can be used to distinguish softwood from hardwood, i.e., the difference between G-type lignin and S-type lignin content (Liu et al. 2004; Yang et al. 2012). The greater the S-type lignin content, the more reddish or reddishpurple will be the stained wood (Nakagawa et al. 2012). The Wiesner staining reaction stains both S-type lignins and G-type lignins in wood cell walls a reddish-purple (Yang et al. 2012). The slightly reddish-purple color of the lignins after the Wiesner staining reaction indicates a greater S-type lignin content, while the dark reddish-purple indicates a greater G-type lignin content (Takabe et al. 1992; Nakagawa et al. 2012; Cui et al. 2016). The lignin content also has been determined via fluorescence reaction. Generally, the greater the fluorescence intensity, the greater was the lignin content $(\mathrm{Xu}$ et al. 2009; Nakagawa et al. 2012; Wang et al. 2012; Liu et al. 2017). Therefore, the authors could judge the lignin content in different cell types and wall structures of the wood cell wall according to the intensity of the fluorescence reaction.

In this study, the Mäule staining reaction, the Weisner staining reaction, and a fluorescence reaction were performed on several common wood species found in the Yunnan province of China. These wood species were qualitatively analyzed to determine the lignin content and obtain data references and a theoretical basis to establish whether these species were suitable for bending and compression treatments or pulping and paper making.

\section{EXPERIMENTAL}

\section{Materials}

The materials selected and tested were samples from multiple wood species: Cunninghamia lanceolate (as control); Tectona grandis with poor compression and bending properties; and Fagus longipetiolata, Betula alnoides, and Fraxinus mandshurica with excellent compression and bending properties. They were purchased from the Kung Ming wood market (Yunnan province, China) with no defects and an air dry moisture content of approximately $10 \%$.

\section{Methods}

\section{Mäule staining reaction}

The Mäule staining reaction was carried out as follows: $10 \mu \mathrm{m}$ to $20 \mu \mathrm{m}$ sections were cut with a microtome (SM2000R, Leica, Buffalo Grove, IL). They were treated with a $1 \%$ potassium permanganate $\left(\mathrm{KMnO}_{4}\right)$ aqueous solution for 5 min, washed 3 times with distilled water, then treated with $3 \mathrm{M}$ hydrochloric acid $(\mathrm{HCl})$ for $1 \mathrm{~min}$, washed again 3 times with distilled water, and then mounted in a $29 \%$ ammonia water solution. The sections were then observed via a light microscope (ECLIPSE 80i, Nikon, Tokyo, Japan) (Takabe et al.1992; Yang et al. 2010, 2012; Liu et al. 2017).

\section{Wiesner staining reaction}

The Wiesner staining reaction was carried out as follows: $10 \mu \mathrm{m}$ to $20 \mu \mathrm{m}$ sections were cut with a microtome and were treated with a $2 \%$ phloroglucin in $95 \%$ ethanol 
solution for $5 \mathrm{~min}$ and then mounted with a $6 \mathrm{M}$ hydrochloric acid $(\mathrm{HCl})$ solution. The sections were then observed via a light microscope (Yang et al. 2010; Nakagawa et al. 2012; Yang et al. 2012; Liu et al. 2017).

\section{Fluorescence reaction}

The fluorescence reaction was carried out as follows: $10 \mu \mathrm{m}$ to $20 \mu \mathrm{m}$ sections were cut with a microtome and were stained with $0.001 \%$ acriflavine and then dehydrated with an ethanol solution with different gradients $(30 \%, 50 \%, 70 \%, 90 \%$, and $100 \%)$ then mounted with a $70 \%$ glycerol solution. The sections were then observed via fluorescence microscope (blue light with 488nm excitation source) (Donaldson et al. 2001; Xu et al. 2009; Nakagawa et al. 2012; Cui et al. 2016; Liu et al. 2017).

\section{Working assumptions regarding the staining assays}

To facilitate interpretation of the experimental findings, two key assumptions were made in this work. The first working hypothesis was that each of the three staining assays was $100 \%$ specific to the type or types of lignin, as described above. Though this assumption goes beyond what has been shown by others (Liu et al. 2004; Yang et al. 2012), it simplifies the interpretation. Second, it was assumed that the depth of staining will be directly proportional to the amount of target compound in the wood, i.e. the concentration of S-lignin, G-lignin, or their sum, in the case of the three assays. Again this assumption goes beyond what is claimed in the prior work (Takabe et al. 1992; Nakagawa et al. 2012; Cui et al. 2016). There is a need for further experimentation to evaluate the precision of the anticipated proportionality. It is reasonable to expect that the depth of staining may depend partly on other factors such as the density of the wood material, details about the microstructure, and whether access to lignin domains are partly blocked by the polysaccharide components of the wood.

\section{RESULTS AND DISCUSSION}

\section{Histochemical Staining Reactions of Cunninghamia lanceolate}

The lignins in softwoods are primarily composed of G-type structure units, while the lignins in hardwoods include G- type and S-type structure units. Figure 1 shows the microstructures of the histochemical staining reactions in wood cell walls for Cunninghamia lanceolate. The cell walls of the tracheids and wood rays after the Mäule staining were stained a light yellowish-brown colour, as shown in Fig. 1a. The results showed that the lignins of the cell walls of the tracheids and wood rays were composed of G-type units, and almost no S-type units. The cell walls of the tracheids and wood rays after the Wiesner staining were stained a purple colour, as shown in Fig. 1b. The wood contained very few S-type lignins, so the purple colour should have come from the G-type lignins. Fluorescence microscopy revealed that the wood had a strong green fluorescence phenomena after undergoing the fluorescence reaction, as shown in Fig. 1c, and indicated that there was a high lignin content in the wood. The greater the lignin content in the wood, the more difficult the wood was to bend or compress. In addition, the cell wall layers of the tracheids and wood rays were observed. It was found that a strong green fluorescence phenomena was found in the compound middle lamellar (CML), the cell corners (CC), and the secondary cell walls (S) of the tracheids and wood rays. Thus, the lignin content in all the tracheid and wood ray wall structures was high. 


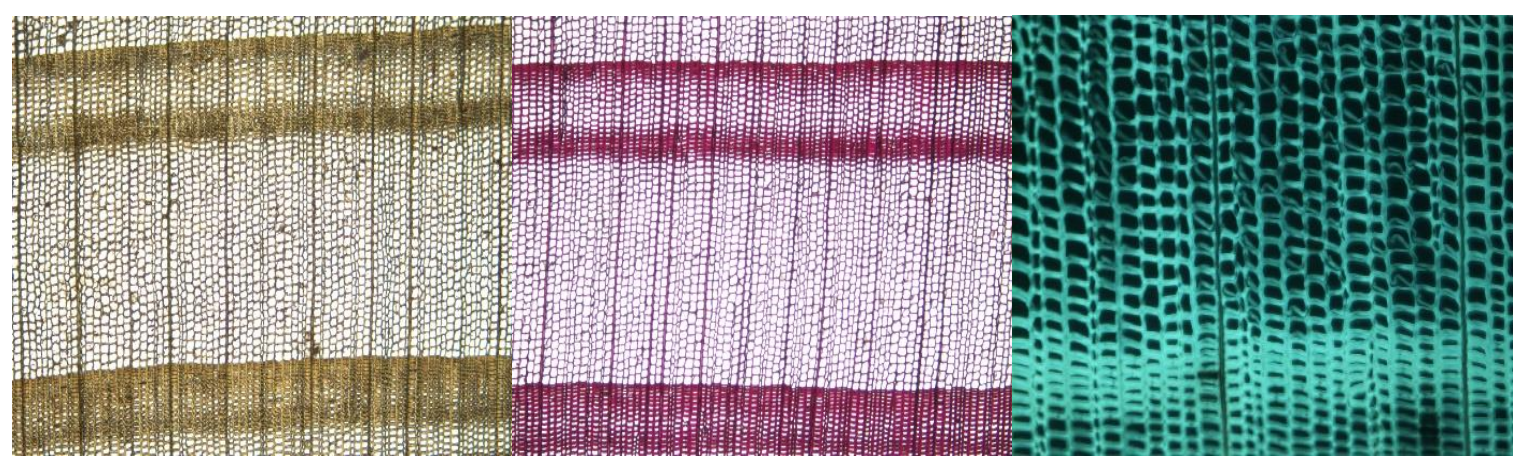

(a) Mäule reaction staining (10X); (b) Miesner staining (10X); and (c) Fluorescence reaction (20X)

Fig. 1. Microstructure images of lignins in Cunninghamia lanceolate after histochemistry stainings

\section{Histochemical Staining Reactions of Betula alnoides}

Figure 2 shows the microstructures after the histochemical staining reactions in the wood cell walls for the Betula alnoides samples. The colour of the cell walls of the vessels, wood fibers, axial parenchymas, and wood rays after the Mäule staining reaction was a deeper red (Fig. 2a) than the Cunninghamia lanceolate samples (Fig. 1a). The results showed that there was a greater S-type lignin content in the vessels, wood fibers, axial parenchymas, and wood rays. Fergus and Goring $(1970 \mathrm{a}, \mathrm{b})$ found that there was a greater G-type lignin content in the cell walls of wood vessels in Betula spp., while a greater Stype lignin content was found in the cell walls of the wood fibers. Musha and Goring (1975) also found that a greater G-type lignin content was found in the cell walls of the wood vessels of 14 woods. The colours of the cell walls of the wood fibers, axial parenchymas, and wood rays after the Wiesner staining reaction were reddish-purple, as shown in Fig. 2b. Combined with the Mäule staining reaction, as shown in Fig. 2a, the Stype lignins were primarily stained via the Wiesner staining reactions to a reddish-purple. Fluorescence microscopy revealed that it had a weaker green fluorescence phenomena after the fluorescence reaction in Betula alnoides, as shown in Fig. 2c, than the fluorescence reaction in Cunninghamia lanceolate. This indicated that there was a lower lignin content in Betula alnoides. Therefore, the difficulties of performing compression and bending processes on this wood species would be decreased, due to the lower lignin content. In addition, the cell wall layers of the wood fibers were observed, and it was found that the brightness was lower for the CML, CC, and S in the cell wall of the wood fibers.

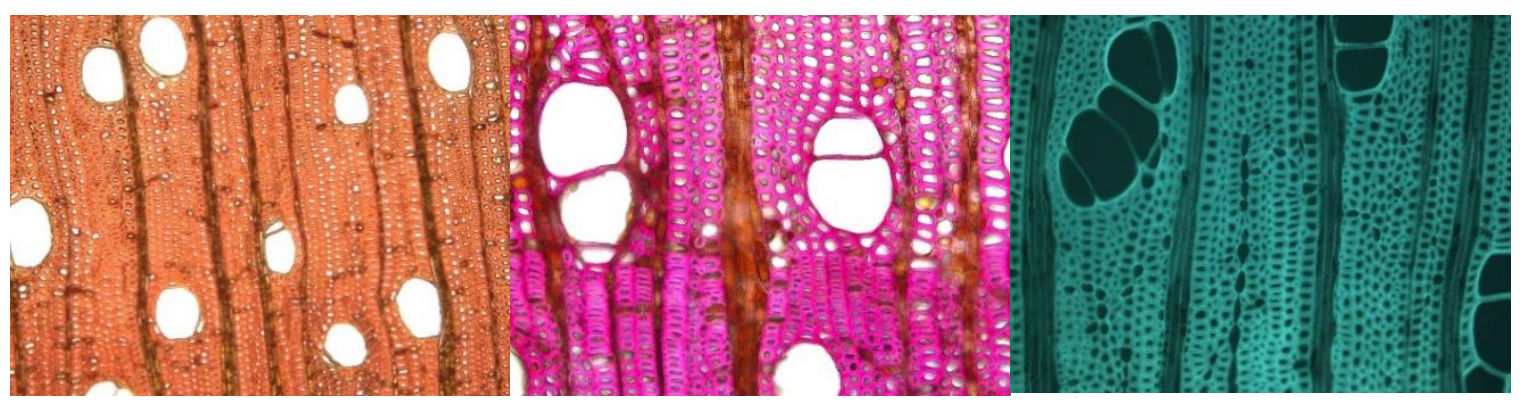

Fig. 2. The microstructure images of the lignins in Betula alnoides after histochemistry stainings. (a) Mäule reaction staining (10X); (b) Miesner staining (10X); and (c) fluorescence reaction (20X) 


\section{Histochemical Staining Reactions of Fagus longipetiolata}

Figure 3 shows the microstructures after the histochemical staining reactions in the wood cell walls for the Fagus longipetiolata samples However, different type cell walls showed different colours. Among them, the colours of the cell walls of the latewood vessels, wood fibers, and axial parenchymas after the Mäule staining were a deep red, while the earlywood vessels and wood rays were a yellowish-brown, as shown in Fig. 3a. The results showed that there was a greater S-type lignin content in the latewood vessels, wood fibers, and axial parenchymas, while a greater G-type lignin content was found in earlywood vessels and wood rays. When the early and late wood fibers subjected to the Mäule staining reactions were compared, it was found that the early wood fibers were more reddish than the late wood fibers, which indicated that the cell walls of the early wood fibers had a greater S-type lignin content than the cell walls of the late wood fibers. Takabe et al. (1992) also found that there was a greater G-type lignin content in the cell walls of the early wood vessels of Fagus crenata than the cells walls in late wood vessels. In addition, the S-type lignin content in the cell walls of early wood fibers was greater than the cell walls of late wood fibers.

The colour of the cell walls of the wood fibers, axial parenchymas, and wood rays after the Wiesner staining reaction were a reddish-purple, as shown in Fig. 3b. Combined with the Mäule staining reaction (as shown in Fig. 3a), both the S-and G-type lignins were stained by the Wiesner staining reactions to a reddish-purple.

Fluorescence microscopy exhibited a weaker green fluorescence phenomena after the fluorescence reaction in Fagus longipetiolata, as shown in Fig. 3c, than the fluorescence reaction in Cunninghamia lanceolate. This indicated that Fagus longipetiolata had a lower lignin content. Therefore, the difficulties of performing compression and bending processes on this wood species would be decreased, due to the lower lignin content. In addition, the cell wall layers of the wood fibers were observed. It was found that the brightness of the CML and CC in the cell walls of the wood fibers were much greater than that of the S, which indicated that the CML and CC in the cell walls of the wood fibers had a greater lignin content than the $\mathrm{S}$ did.

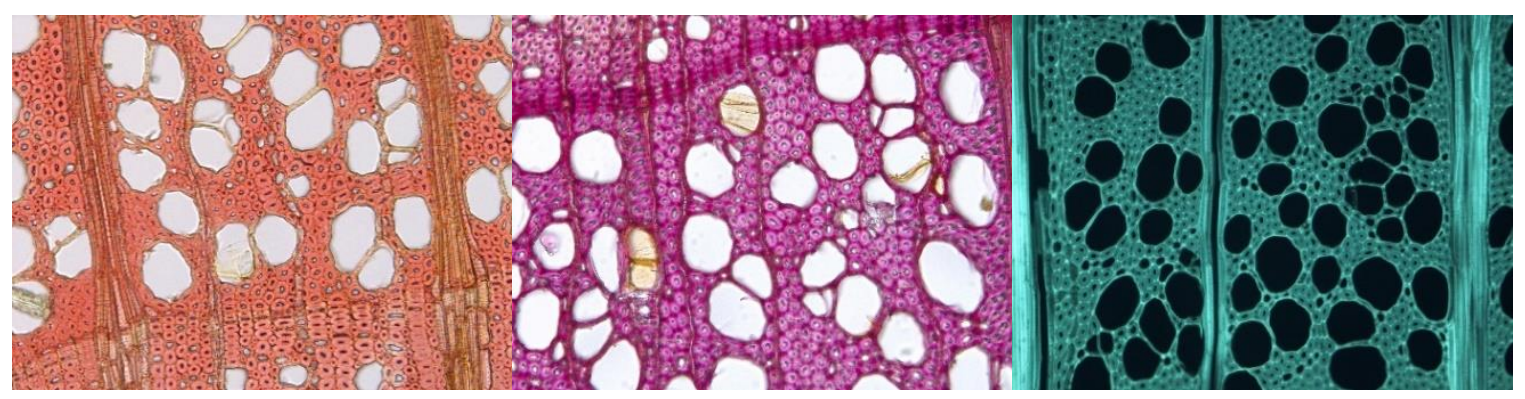

Fig. 3. The microstructure images of the lignins in Fagus longipetiolata after histochemistry stainings. (a) Mäule reaction staining (10X); (b) Miesner staining (10X); and (c) Fluorescence reaction $(20 \mathrm{X})$

\section{Histochemical Staining Reactions of Fraxinus mandshurica}

Figure 4 showed the microstructures after the histochemical staining reactions in the wood cell walls for the Fraxinus mandshurica samples. The colours of the cell walls of the vessels, wood fibers, axial parenchymas, and wood rays after the Mäule staining were a deeper red (Fig. 4a) than the cell walls of Cunninghamia lanceolate (Fig. 1a). The results showed that there was a greater S-type lignin content compared to the other lignin 
types in the vessels, wood fibers, axial parenchymas, and wood rays. The colour of the cell walls of the vessels, fibers, axial parenchymas, and wood rays after the Wiesner staining reaction was a reddish-purple, as shown in Fig. 4b. When combined with the Mäule staining reaction (Fig. 4a), the S-type lignins in the vessels, fibers, axial parenchymas, and wood rays after also being stained by the Wiesner staining reactions turned to a reddishpurple. Fluorescence microscopy revealed that it had a weaker green fluorescence phenomena after the fluorescence reaction in Fraxinus mandshurica (Fig. 4c) than the reaction in Cunninghamia lanceolate (Fig. 1c), but had a stronger green fluorescence phenomena than the fluorescence reactions of Betula alnoides (Fig. 2c) and Fagus longipetiolata (Fig. 3c). The brightness of the vessels and axial parenchymas were stronger than the wood rays and fibers, which indicated that the cell walls of the vessels and axial parenchymas had a greater lignin content than the wood rays and fibers. In addition, the cell wall layers of the vessels and axial parenchymas were observed. It was found that the CML, CC, and S layers all showed high brightness, which indicated that the CML, CC, and S layer in the cell walls of the vessels and axial parenchymas had a greater lignin content.

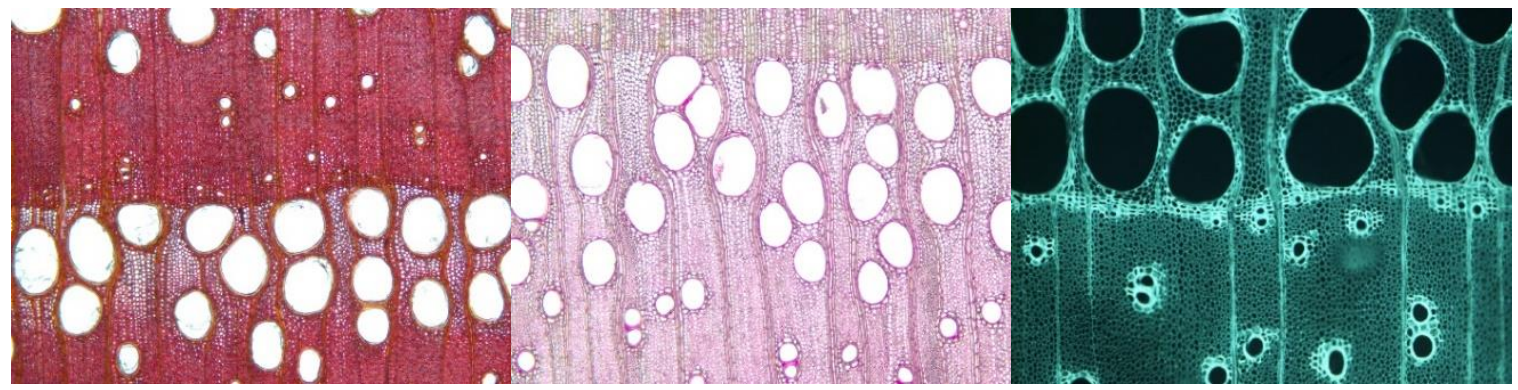

Fig. 4. The microstructure images of the lignins in Fraxinus mandshurica after histochemistry stainings. (a) Mäule reaction staining (10X); (b) Miesner staining (10X); and (c) Fluorescence reaction (20X)

\section{Histochemical Staining Reaction of Tectona grandis}

Figure 5 shows the microstructures after the histochemical staining reactions in the wood cell walls for the Tectona grandis samples. The colour of the cell walls of the vessels, wood fibers, axial parenchymas, and wood rays after the Mäule staining were a deeper yellowish-brown (Fig. 5a and 5b). This was observed especially for the colour of the tangential section (Fig. 5b), which was similar to the color of the Cunninghamia lanceolate samples after the Mäule staining (Fig. 1a). The results showed that there was a greater Gtype lignin content compared to the other lignin types in the cell walls of the vessels, wood fibers, axial parenchymas, and wood rays in Tectona grandis. The colour of the cell walls of the vessels, fibers, axial parenchymas, and wood rays after the Wiesner staining reaction were a reddish-purple, as shown in Fig. 5c. When combined with the Mäule staining reaction (Fig. 5a and 5b), the G-type lignins in the vessels, fibers, axial parenchymas, and wood rays were stained via the Wiesner staining reactions to a reddish-purple. Fluorescence microscopy revealed that it had a stronger green fluorescence phenomena after the fluorescence reaction in Tectona grandis (Fig. 5d) than the fluorescence reactions in Betula alnoides (Fig. 2c), Fagus longipetiolata (Fig. 3c), and Fraxinus mandshurica (Fig. 4c), but it had a similar reaction to Cunninghamia lanceolate (Fig. 1c). 


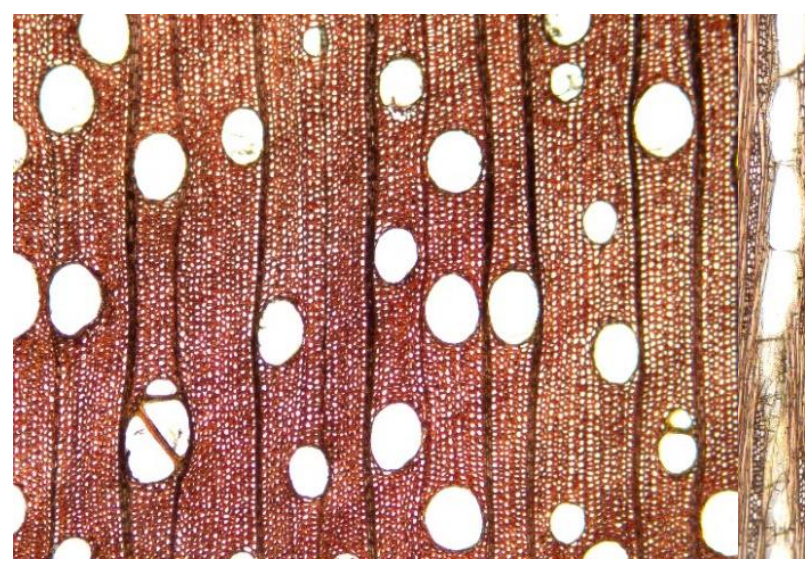

(a) Mäule reaction staining (10X)

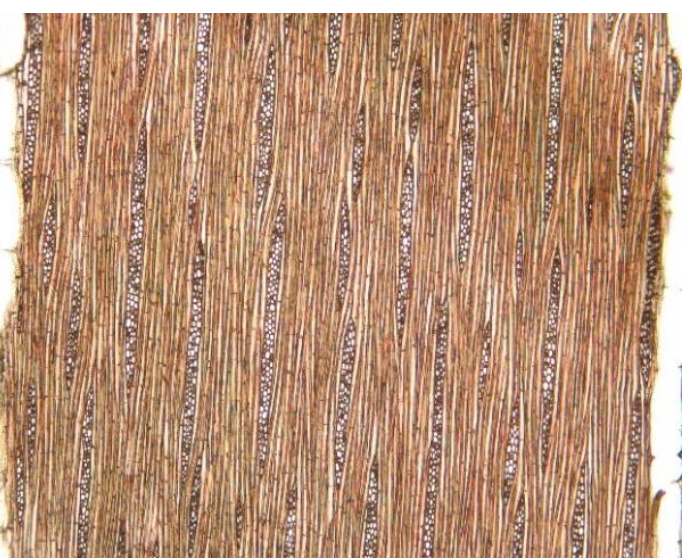

(b) Mäule reaction staining (10X)

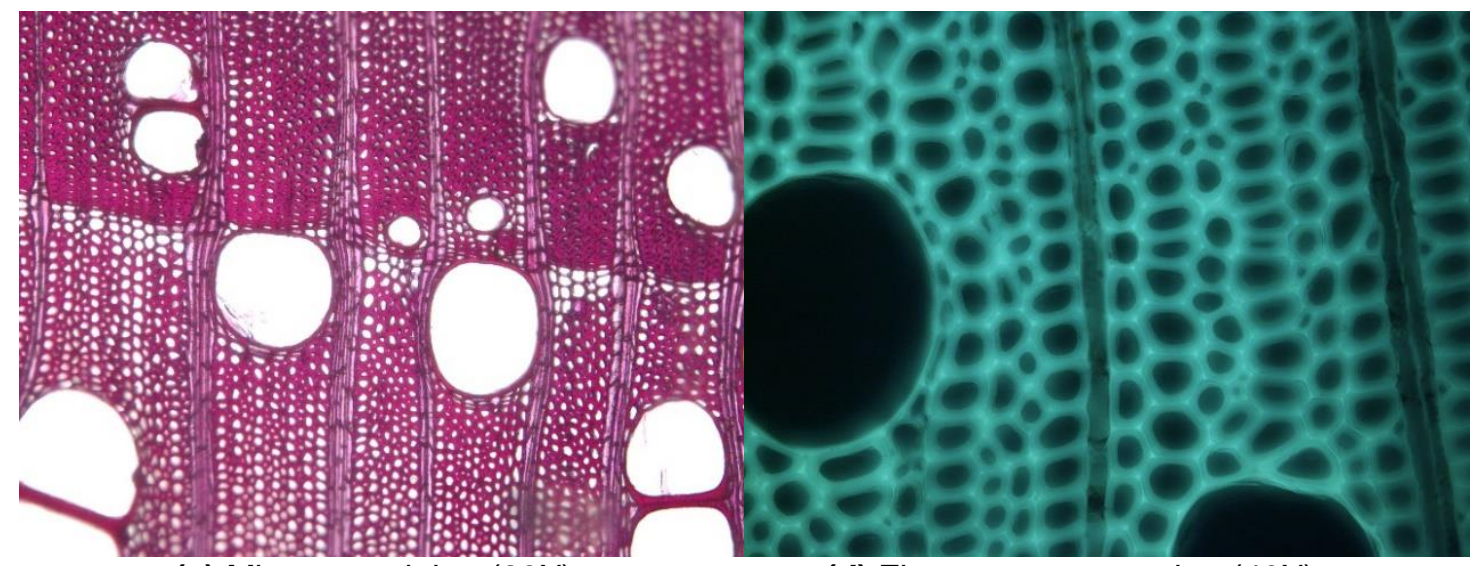

(c) Miesner staining (20X)

(d) Fluorescence reaction (40X)

Fig. 5. The microstructure images of the lignins in Tectona grandis after histochemistry stainings.

Some scholars have used a chemical analysis method to determine the lignin content of the above-mentioned woods. The lignin content of a branch of Fraxinus rhynchophylla was 26\% (Nie 2012), Betula alnoides had a lignin content of 23.69\% (Yang et al. 2015; Yang et al. 2016), while the lignin content of Tectona grandis was greater than 30.91\% (Moya et al. 2010; Miranda et al. 2011; Lourenço et al. 2015). The lignin content of Tectona grandis was also determined by the authors in this paper and the result was consistent with the results of Moya et al. (2010), Miranda et al. (2011), and Lourenço et al. (2015).

The authors in this paper found that the bending property of Tectona grandis (the ratio of the thickness of the curved wood $(t)$ to the minimum radius of the curvature $(r)$ was used as the evaluation index, where $t / r$ was less than $1 / 8$, the datum was unpublished) was much lower than the bending property of Fraxinus rhynchophylla $(t / r=1 / 2)$ (Shen et al. 2000). The greater the lignin content, the greater the rigidity. Therefore, the bending and compression properties of Tectona grandis were poor. There was also evidence that Ulmus davidiana var. japonica $(t / r=1 / 3)$ (Lu et al. 2013) and Sassafras tzumu $(t / r=1 / 5)$ (Yao 2014) also had good bending properties 


\section{CONCLUSIONS}

1. The lignin type in Cunninghamia lanceolate was the G-type, and the fluorescence reaction was very strong, which implied that there was a greater lignin content in this wood. The greater lignin content would increase the difficulty of performing compression and bending processes on this wood species.

2. The fluorescence reactions to the lignins in the wood of Betula alnoides, Fagus longipetiolata, and Fraxinus mandshurica were not obvious, which indicated that the lignin contents in the woods was lower than the other tested species. The lower lignin content would reduce the difficulty of performing compression and bending processes on these wood species.

3. Tectona grandis contained more G-type lignins, and the overall fluorescence reaction was stronger than other tested species, which indicated that the wood had a greater lignin content. The greater lignin content would increase the difficulty of performing compression and bending processes on this wood species.

4. There was a negative correlation between the lignin content in the wood cell walls and the bending and compression properties of the woods. Therefore, the application of histochemical stains for the analysis of wood lignin content could provide a reference and experimental basis for whether they would be suitable for bending or compression treatments of various wood species.

\section{ACKNOWLEDGMENTS}

The authors are grateful for the supports of Natural National Science Foundation of China (31700481), Cross-science Research Project of Nanyang Institute of Technology (presented by Yan YANG), Scientific Research Start-up Projects of Nanyang Institute of Technology (presented by Yan YANG and Wei WANG) and the dominant characteristic disciplines of Nanyang Institute of Technology (presented by Jianwen ZHANG ).

\section{REFERENCES CITED}

Cui, H.-S., Yang, S.-M., Liu, X.-E., Ma, J.-F., and Tian, G.-L. (2016). "Chemical composition and lignin distribution of Salix integra," Chemistry and Industry of Forest Products 36(5), 120-126. DOI: 10.3969/j.issn.0253-2417.2016.05.018

Donaldson, L., Hague, J., and Snell, R. (2001). "Lignin distribution in coppice poplar, linseed and wheat straw," Holzforschung 55(4), 379-385. DOI:

10.1515/HF.2001.063

Fergus, B. J., and Goring, D. A. I. (1970a). "The location of guaiacyl and syringyl lignins in birch xylem tissue," Holzforschung 24(4), 113-117. DOI:

10.1515/hfsg.1970.24.4.113

Fergus, B. J., and Goring, D. A. I. (1970b). "The distribution of lignin in birch wood as determined by ultraviolet microscopy," Holzforschung 24(4), 118-124. DOI:

10.1515/hfsg.1970.24.4.118 
Liu, X.-E, Jin, K.X., Cui, H.-S., and Ma, J.-F. (2017). "The lignin topochemistry of Daemonorops margaritae (Hance) Becc. by molecular spectroscopic imaging," Spectroscopy and Spectral Analysis 37(10), 3138-3144.

Liu, Y.-X., and Zhao, G.-J. (2004). Wood Resource Material Science, Chinese Forestry Press, Beijing, China.

Liu, C.-W., Su, M.-L., Zhou, X.-W., Zhao, R.-J., Lu, J.-X., and Wang, Y.-R. (2017). "Analysis of content and distribution of lignin in cell wall of transgenic poplar with Fourier infrared spectrum (FTIR) and confocal laser scanning microscopy (CLSM)," Spectroscopy and Spectral Analysis 37(11), 3404-3408.

Lourenço, A., Neiva, D. M., Gominho, J., Marques, A. V., and Pereira, H. (2015). "Characterization of lignin in heartwood, sapwood and bark from Tectona grandis using Py-GC-MS/FID," Wood Science and Technology 49(1), 159-175. DOI: 10.1007/s00226-014-0684-6

Lu, X.-J, Liu, D.-F, and Song, K.-Y. (2013). "Study on the bending performance of hydrothermal-treated elm wood after longitudinal compression," Forestry Machinery \& Woodworking Equipment 41(8), 41-43.

Miranda, I., Sousa, V., and Pereira, H. (2011). "Wood properties of teak (Tectona grandis) from a mature unmanaged stand in East Timor," Journal of Wood Science 57(3), 171-178. DOI: 10.1007/s10086-010-1164-8

Musha, Y., and Goring, D. A. I. (1975). "Distribution of syringyl and guaiacyl moieties in hardwoods as indicated by ultraviolet microscopy," Wood Science and Technology 9(1), 45-58. DOI: 10.1007/BF00351914

Nakagawa, K., Yoshinaga, A., and Takabe, K. (2012). “Anatomy and lignin distribution in reaction phloem fibres of several Japanese hardwoods," Annals of Botany 110(4) 897-904. DOI: $10.1093 / \mathrm{aob} / \mathrm{mcs} 144$

Nie, M.-F. (2012). "A study of growth ring eccentricity in North American white ash branch wood," Quality and Technical Supervision Research 24(6), 24-27.

Shen, D.-J., Sui Z.-H., and Fang H.-T. (2000). "On the study of crooking ability for Fraxinus rhynchophylla Hance," Journal of Jilin Forestry University 16(4), 204-205.

Takabe, K., Miyauchi, S., Tsunoda, R., and Fukazawa, K. (1992). "Distribution of guaiacyl and syringyl lignins in Japanese beech (Fagus crenata): Variation within an annual ring," IAWA Journal 13(1), 105-112. DOI: 10.1163/22941932-90000561

Wang, Y.-R., Xing, X.-T., Ren, H.-Q., Yu, Y., and Fei, B.-H. (2012). "Distribution of lignin in Chinese fir branches determined by ultraviolet microspectrometer," Spectroscopy and Spectral Analysis 32(6), 1685-1688. DOI: 10.3964/j.issn.10000593(2012)06-1685-04

Xu, F., Mao, J.-Z, Jones, G.-L., and Sun, R.-C. (2009). "Ultrastructure and lignin distribution in fiber cell walls of Caragana korshinskii normal wood and tension wood," Transactions of China Pulp and Paper 24(4), 15-18.

Yang Y. (2016). Studies on the Law of Heat and Mass Transfer in Wood and the Color Control During the Thermo-Vacuum Treatment, Ph.D. Dissertation, Chinese Academy of Forestry, Beijing, China.

Yang, S.-M., Liu , X.-E., Fei, B.-H., Jiang, Z.-H., Yang, X., and Shan, H.-B. ( 2012) . "Main anatomy characteristics in cell wall and lignin distribution of bamboo culms (Pseudosasa amabilis)," Scientia silvae sinicae 48(2),129-134. DOI:

10.11707/j.1001-7488.20120219 
Yang, S.-M., Ren, H.-Q, Fei, B.-H., and Jiang, Z.-H. (2010). "Lignin distribution in cell wall of bamboo culms (Phyllostachys pubescens and Pseudosasa amabilis)," Chemistry and Industry of Forest Products 30(5), 19-24.

Yang, Y., Zhan, T. Y., Lu, J. X., and Jiang, J. H. (2015). "Influences of thermo-vacuum treatment on colors and chemical compositions of alder birch wood," BioResources 10(4), 7936-7945. DOI: 10.15376/biores.10.4.7936-7945

Yao, W.-L. (2014). Bending Process of Catalpa, Ph.D. Dissertation, Central South University of Forestry and Technology, Changsha, China.

Article submitted: October 10, 2019; Peer review completed: January 23, 2020; Revised version received: January 29, 2020; Accepted: February 3, 2020; Published: March 30, 2020.

DOI: 10.15376/biores.15.2.3524-3533 